\title{
Dynamic Tensile Response of Caprine Muscles Using Split Hopkinson Pressure Bar
}

\author{
Somnath H. Kadhane*, Hemant N. Warhatkar \\ Department of Mechanical Engineering, Dr. B. A. Technological University, Lonere-Raigad, 402103, Maharashtra State, India
}

Received March 6, 2020; Revised June 22, 2020; Accepted July 1, 2020

\section{Cite This Paper in the following Citation Styles}

(a): [1] Somnath H. Kadhane, Hemant N. Warhatkar, "Dynamic Tensile Response of Caprine Muscles Using Split Hopkinson Pressure Bar," Universal Journal of Mechanical Engineering, Vol. 9, No. 1, pp. 1 - 9, 2021. DOI: 10.13189/ujme.2021.090101.

(b): Somnath H. Kadhane, Hemant N. Warhatkar (2021). Dynamic Tensile Response of Caprine Muscles Using Split Hopkinson Pressure Bar. Universal Journal of Mechanical Engineering, 9(1), 1 - 9. DOI: 10.13189/ujme.2021.090101.

Copyright $\subset 2021$ by authors, all rights reserved. Authors agree that this article remains permanently open access under the terms of the Creative Commons Attribution License 4.0 International License

\begin{abstract}
Computer modeling and numerical simulation has become an efficient diagnostic tool to predict the human body injuries caused due to high speed automotive impacts, blast and ballistic impacts. Soft tissues such as muscles and skin in human body are exposed to varying strain rates under dynamic loadings during impacts. The prediction of impact-induced injuries requires a thorough understanding of mechanical behaviour of soft tissues for computational modeling of human body. In the present study, uniaxial tensile tests were conducted on caprine lower extremity muscles in the strain rate range of $\left(500 \mathrm{~s}^{-1}-3500 \mathrm{~s}^{-1}\right)$ using custom-made split Hopkinson pressure bar (SHPB) apparatus. The challenges in the dynamic testing of soft tissues such as measurement of weak transmitted signals, use of viscoelastic pressure bars, tensile loading of specimen and generation of constant strain rate were addressed in dynamic tensile testing of soft tissues using polymeric SHPB. The attenuation and dispersion in waves are corrected using isolated incident bar tests. The stress-strain results were determined from the reconstructed waves for the tests conducted on lower extremity caprine muscles. The muscle specimens were tested along and perpendicular to the fiber direction to study the directional dependency of tissue behaviour. The stress-strain response was found to be non-linear and significant dependant on strain rate when tested along and perpendicular to fiber direction at same strain rates. It is also observed that at the same strain rate, the specimen stress of caprine muscle along the perpendicular fiber direction is higher than that along the fiber direction. The obtained results may further be used to develop finite
\end{abstract}

element human body models and safety systems for human body in high rate scenario.

Keywords Tensile Loading, SHPB, Strain Rate, Impact, Tissue Behaviour, Caprine Muscle

\section{Introduction}

Higher strain rates occur not only in automobile impacts, but also in airplane accidents, impact or bird-strike events, sport accidents, industrial accidents, slip and falls, explosive blast, ballistic and bullet impacts. A high strain rate response of muscle tissue is essential for computer modeling of soft tissue in research and application activities of impact biomechanics. The mechanical response of soft biological tissues at different strain rates is also essential in high speed automotive impacts and crash analysis to develop human body models and to design body armors for effective understanding of the injury mechanisms. Lower extremity muscles of human body are subjected to compressive impact during pedestrian-vehicle impacts. The muscle constitutes are compressed in the direction transverse to the fiber orientation and slide over each other, while the muscle constitutes are stretched in the direction parallel to the fiber orientation. Since muscles are formed of fiber bundles, the tensile behavior will be different than the compressive behavior.

The available quantitative data on the mechanical response of biological soft tissues is mostly limited to 
quasi-static strain rates $\left(\dot{\varepsilon}<0.1 \mathrm{~s}^{-1}\right)$ [1-4]. The response of soft tissues at intermediate strain rates $\left(\dot{\varepsilon}>5 \mathrm{~s}^{-1}\right)$ have also been studied for bovine medial collateral ligament [2], human muscle [4], human spleen [5], human diaphragm [6] and human aortic tissue [7] under tensile loading. The response of soft tissues at intermediate strain rates $\left(\dot{\varepsilon}>5 \mathrm{~s}^{-1}\right)$ have been studied for skeletal muscle [1], porcine muscle [3], bovine liver tissue [14] and adipose tissue [17] under compressive loading. Studies on muscle tissue responses under dynamic loadings at higher strain rates are limited in available literature. SHPB, originally developed by Hopkinson [89] has been critically studied by Davis [10] and later improved by Kolsky [11] with two pressure bars connected in series. SHPB has been widely used to characterize the dynamic behavior of ductile materials in the strain rate range of $10^{2}-10^{4} \mathrm{~s}^{-1}$ [12]. This technique has been modified for testing soft tissues under compressive, tensile and torsional loading. A few research attempts have been invested in the dynamic characterization of soft tissues at higher strain rates due to experimental issues in the characterization of soft tissues. Over a decade, SHPB with low impedance metallic bars has been widely used to investigate the dynamic stress-strain response of soft tissues under compressive loading and such response has been found to very different form the quasi-static response. The compressive stress-strain responses of bovine soft tissues such as kidney tissue [13], liver tissue [14], brain tissue [15]; porcine soft tissues such as liver tissue [16], adipose tissue [17], trachea tissue [18] and lung tissue [19] have been recently studied over wide range of strain rates using modified versions of low impedance metallic SHPB. The compressive mechanical response of porcine muscle [20] and bovine muscle [21] using low impedance metallic bars and PMHS muscle [22] and goat muscle [23] using polymeric SHPB has been studied systematically at high strain rates. The experimental results reveals that the soft tissue responses were non-linear and dependant on strain rate.

Experiments to obtain the mechanical response of soft tissues at high strain rates under tensile loading have been limited and less tensile data is reported in literature as it is very challenging to subject the soft tissue specimen to valid dynamic loading conditions and measure their mechanical responses. The tensile stress-strain responses of porcine muscle [24-25], bovine tendon [26], pig skin [27-28] and human cervical spine ligaments [29] have been recently studied over a wide range of strain rates using modified versions of SHPB. Experiments of soft tissue responses under tension are scarce [24-29]. An accurate and realistic material model that represents the dynamic response of soft tissues requires more data on tensile response of soft tissues at high strain rates. The shear response of bovine brain tissue [30] and porcine lung tissue [19] has also been studied under torsional loading using modified SHPB with shear grip attachment. The porcine muscles were tested at a strain rate range of $700-2100 \mathrm{~s}^{-1}$ [24], the bovine tendons were tested at a strain rate of $2500 \mathrm{~s}^{-1}$ [26] and the pig skin tissues were tested in the strain rate range of $1700-3500 \mathrm{~s}^{-1}$ [28].

The major challenges in characterization of soft tissues are low signal to noise ratio, use of low impedance pressure bars, the attainment of dynamic stress equilibrium and constant strain rate in specimen and generation of low amplitude repeated incident pulse. The remedial solutions to these challenges as suggested in literature [31] has been incorporated in custom-made polymeric SHPB for tensile testing of soft tissue. The weak signals are propagated through transmission bar during soft tissue testing using metallic SHPB due to acoustical impedance and geometrical mismatch of pressure bars and specimen. This can be improved by using low-impedance metallic bars such as titanium, aluminum and magnesium alloy bars and hollow transmission bar. The use of hollow transmission bar introduces uncertainty in readings as signals propagating through solid bar and hollow bar are not same. The polymeric SHPB enables the measurement of low-stress signals and allows the inspection of stress-equilibrium in the test specimen. The specimen is subjected to longer test time due to the lower sound velocities as compared to the low-impedance metallic bars, and thus enables large strains applied before the effects of reflected stress-waves. This gives the uniform deformation of the specimen. The benefits of polymeric SHPB are high quality signals may be measured on the pressure bars due to impedance matching between the pressure bars and soft tissues so that and flexibility in specimen geometry due to longer stress wave rise time for the PMMA material. The elastic analysis of SHPB cannot be directly applied for polymeric bars as polymeric bars are viscoelastic in nature. The stress wave attenuates and disperses in the medium due to the use of polymeric bars in SHPB. The strain measured at the middle location of the pressure bar is different from the strain measured at the interface of bar-specimen.

In the present study, the challenges in the dynamic testing of soft tissues such as measurement of weak transmitted signals, use of viscoelastic pressure bars, tensile loading of specimen and generation of constant strain rate were addressed in dynamic tensile testing of soft tissues using polymeric SHPB. We used polymeric pressure bars to obtain dynamic stress-strain response of caprine muscle tissues and wave correction was applied. The Labview assisted data acquisition system was used to acquire the stress waves propagating through the pressure bars during impact. Matlab program based on viscoelastic analysis of SHPB was used for dynamic characterization of muscle tissues. Polymeric SHPB setup and Matlab program has been validated. The experimental protocol in dynamic testing of soft tissues has been adopted from previous studies [22-24]; however the protocol to prepare the caprine muscles specimens for tensile testing was developed in-house. The muscle specimens were tested 
along and perpendicular to the fiber direction to study the directional dependency of tissue behaviour. The stress-strain response of caprine muscle tissues along and perpendicular to muscle fiber direction is obtained by using custom-made polymeric SHPB under tensile loading at varying strain rates. Experimental tests at higher loading rates of $400-3700 \mathrm{~s}^{-1}$ are necessary to understand the tissue behavior and injury patterns not only during automobile accidents but also during blast or ballistic impacts. During military operations, war-fighters are subjected to blast events. In response to this, a bulletproof jacket is an important protection for the human body from high velocity, short duration blunt and ballistic impacts.

\section{Materials and Methods}

\subsection{Specimen Preparation}

The preparation of specimen and gripping method to avoid the failure of soft tissue at the grips is also a challenging task. This motivates the study on muscle tissue characterization using modified SHPB with polymeric pressure bars. The fresh muscle specimens from lower extremity of three Indian adult goats were collected from a slaughter house. All the specimens were stored in saline solution at room temperature about $20^{\circ} \mathrm{C}$ for three hours before testing. All the specimens were thawed at room temperature for less than three hours before testing. The fascia layer was removed from the muscles. The measures were taken to prevent the dehydration of the muscle tissue during experimentation. Specimens were not preconditioned prior to testing. The physical loading of muscle tissue was avoided till performing the tests.

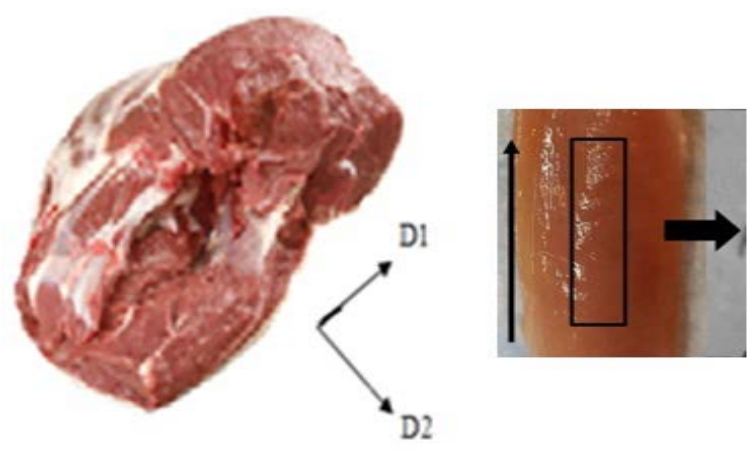

Figure 1. Thin muscle sheet preparation

The preparation of muscle sheet for tensile specimen is represented in figure 1 . The flat muscle sheet is used to prepare a thin tubular specimen for reducing the radial inertia effects produced during axial acceleration of specimen [30, 35]. The muscles were sliced into $3 \mathrm{~mm}$ thick flat sheets along and perpendicular to the fiber orientation of muscle tissue. The width (horizontal arrow) and length (vertical arrow) of the flat sheet is maintained as $30 \mathrm{~mm}$ and $50.3 \mathrm{~mm}$. The width of the muscular sheet is prepared along $\left(\mathrm{D}_{1}\right)$ and perpendicular $\left(\mathrm{D}_{2}\right)$ to the muscle fiber direction. The muscle flat sheets must be then slightly extended and smoothed when they are wrapped around the test section and clamped at both ends of the polymeric pressure bars in order to avoid the fibers of muscle to wrinkle. In order to load the muscle along and perpendicular to the fiber direction, the cylindrical axis of the tubular muscle specimen is kept along the fiber direction and perpendicular to the muscle fiber direction. The cylindrical axis of specimen is the direction of tensile loading during the tensile tests.

\subsection{Specimen Gripping}

The flat muscle sheets are then wrapped around the test section with predetermined gap between incident and transmission bars. The predetermined gap of $1 \mathrm{~mm}$ obtained after trial experiments is used to clamp the muscle sheet around the pressure bar to facilitate axial stress equilibrium. This predetermined gap between the pressure bars acts as width of the specimen and is maintained during every tests by inserting the spacer of $1 \mathrm{~mm}$ thick between the incident and transmitter bars before the wrapping of the specimen. Flat muscle sheet is used as tubular specimen to reduce radial inertia effects. The internal surface of tubular specimen was bonded to cylindrical surface of pressure bar, whereas the outer surface of tubular specimen was clamped at both ends using plastic hose pipe / snap clamps. An illustration of the tubular muscle specimen mounted in the test section is shown in figure 2 .

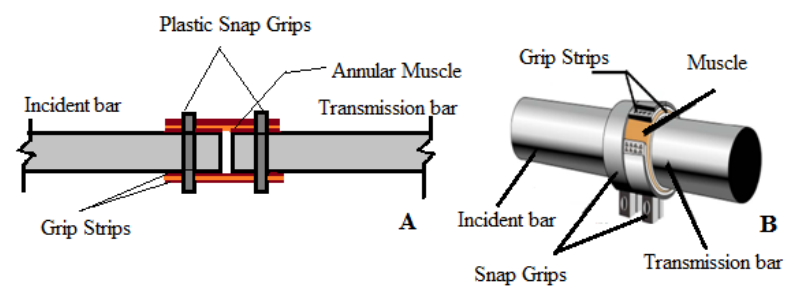

Figure 2. A thin annular muscle specimen mounted between pressure bars for tensile tests (A) 2-D view and (B) 3-D view

The reflected pulse in the incident bar might be affected due to the use of the clamps for mounting of the specimen in between pressure bars under tensile loading. However, the reflected pulse is affected very less and its effect is negligible due the use of plastic hose clamps [00]. The flat sheet was slightly stretched to avoid the wrinkling of muscle fibers while attaching the specimen on pressure bar ends. However, the effect of stretching of specimen and the discontinuity at junction point where the flat muscle sheet meets itself to form tubular geometry of specimen are not studied. This is a limitation of the present study.

\subsection{Experimental Setup}

The schematic of tensile SHPB is shown in figure 3 consists of incident bar, transmission bar and hollow striker bar. All the pressure bars were made from PMMA 
(Poly-methyl Metha-acrylate). The incident and transmitter pressure bars has diameter $16 \mathrm{~mm}$ and length $1220 \mathrm{~mm}$. The hollow striker bar with internal diameter of $16 \mathrm{~mm}$ and external diameter of $32 \mathrm{~mm}$ has been used as projectile to generate for tensile loading of the specimen during experimental tests. The tubular specimen is clamped with predetermined gap between incident and transmitter bar by using plastic snap clamps and the incident stress pulse is generated by the impact of hollow striker bar on the collar attached to the incident bar. The incident and reflected signals were measured by a pair of resistive foil strain gauges (Omega Engineering) mounted the incident bar and transmission bar at in the middle. The velocity of striker bar projectile is controlled by pressurized compressed air that is forced into the launcher chamber.

PTFE (Polytetrafluoroethylene) thin disks as pulse shapers were used to obtain the dynamic stress equilibrium within the specimen. When fired using quick acting solenoid valve, the launcher releases the air and propels a striker bar into the end of incident bar. The collision of striker bar with the collar at the end of incident bar creates the incident pulse (tensile) that propagates through the incident bar towards the specimen. The incident pulse is recorded by the strain gauges mounted on the incident bar. Once the wave reaches to the specimen, it splits into two pulses viz. transmitted wave and reflected wave. The transmitted pulse (tensile) travels through the specimen and into the transmitter bar where the energy is recorded by the strain gauges mounted on transmitter bar. The second pulse (compressive) is reflected away from the specimen and travels back down to the incident bar. At the end of SHPB, a stop bar absorbs the impact of transmission bar to complete the test.

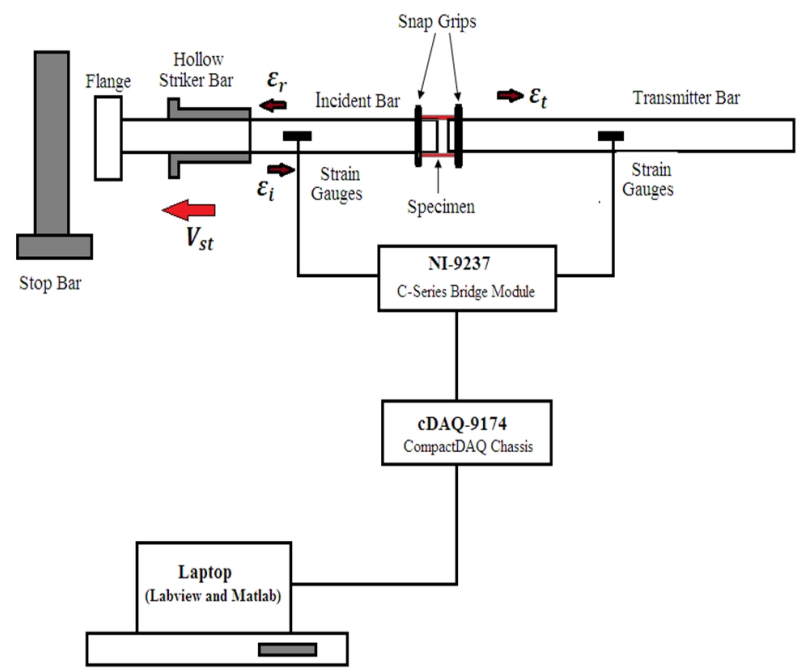

Figure 3. Schematic of tensile polymeric SHPB setup
One dimensional wave propagation theory in elastic pressure bars along axial direction yields the calculation of strain $\left(\varepsilon_{s}\right)$ strain rate $\left(\dot{\varepsilon}_{s}\right)$ and stress $\left(\sigma_{s}\right)$ induced in the specimen using following equations.

$$
\begin{gathered}
\varepsilon_{S}=\frac{-2 C_{0}}{L_{S}} \int_{0}^{t} \varepsilon_{r} d t \\
\dot{\varepsilon}_{S}=\frac{-2 C_{0}}{L_{S}} \varepsilon_{r} \\
\sigma_{S}=\frac{A_{0}}{A_{S}} E \varepsilon_{t}
\end{gathered}
$$

Where, $\varepsilon_{r}$ and $\varepsilon_{t}$ are the reflected and transmitted strain histories, respectively; $A_{0}$ is the cross-sectional area or pressure bars; $C_{0}$ and $E$ are the sound wave speed and modulus of elasticity in the pressure bar, respectively; $A_{S}$ and $L_{S}$ are the initial cross-sectional area and length of specimen, respectively.

An experimental method suggested by Bacon [32] has been applied to account the strain mismatch for the correction of wave attenuation and dispersion. The propagation coefficients obtained from free end tests on the incident bar at $2 \mathrm{~m} / \mathrm{s}$ using the viscoelastic analysis of polymeric SHPB has been used in muscle tissue characterization. The attenuation coefficient and wave number were determined using amplitude and phase spectrum of incident and reflected pulses respectively. The wave numbers at different frequencies were used to find the phase velocities. The reflected pulse and transmitted pulse is reconstructed by incorporating the attenuation and dispersion correction in the respective measured pulses for the distance it has propagated. The calculation of specimen stress, strain and strain rate in the frequency domain was done using viscoelastic analysis for polymeric bars, where the phase velocity is a function of frequency. Stress $\tilde{\sigma}_{S}(\omega)$, strain, $\varepsilon_{S}(\omega)$ and strain rate, $\dot{\varepsilon}_{S}(\omega)$ in the specimen are calculated using reconstructed waves in frequency domain.

$$
\begin{gathered}
\dot{\varepsilon}_{S}(\omega)=-\frac{2 \tilde{\varepsilon}_{R} \_n e w}{L_{0}}(\omega)\left[\frac{i \omega}{\gamma(\omega)}\right] \\
\varepsilon_{S}(\omega)=\int_{0}^{t} \dot{\varepsilon}_{S}(\omega) d t \\
\tilde{\sigma}_{S}(\omega)=\frac{A_{0} \tilde{\varepsilon}_{T} \text { new }(\omega)}{A_{S}} E^{*}(\omega)
\end{gathered}
$$

Using inverse Fourier transform, stress $\sigma_{S}(\omega)$, strain $\varepsilon_{S}(t)$ and strain rate $\dot{\varepsilon}_{S}(t)$ the specimen are then calculated in time domain. 


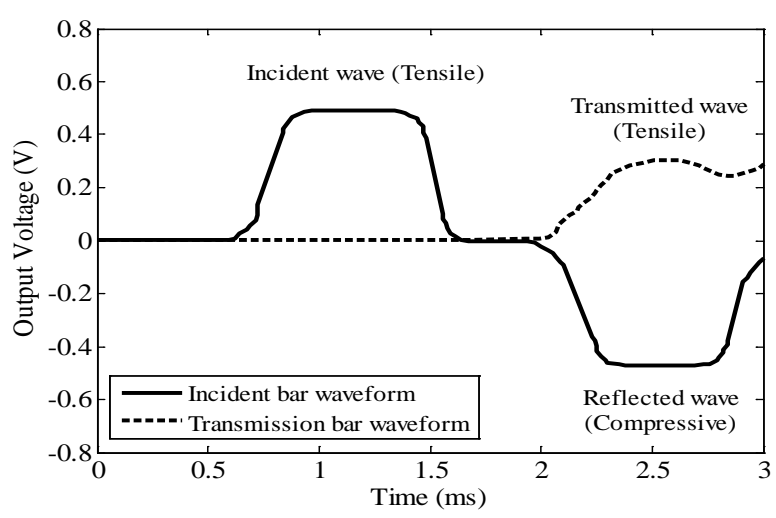

Figure 4. Waveform history during tensile experiments on tubular caprine muscle specimen using polymeric SHPB

The incident, reflected and transmitted stress pulses during tensile experiments on caprine muscle specimen at higher strain rates are shown in figure 4.The frequency oscillations and initial spikes observed in measured stress pulses during experiments using elastic SHPB are nearly eliminated in viscoelastic SHPB tests due to use of pulse shaping and polymeric pressure bars. This facilitates accurate characterization of stress-strain response at higher strain rates under dynamic loading. The incident and reflected pulse are nearly same and opposite in sign due the mechanical impendence of the soft tissue is closer to the pressure bars. The transmitted pulse records the stress history and reflected pulse records the strain history in the specimen. The reflected pulses recorded by incident strain gauges and the portion of compressive pulses that continues through the specimen recorded by transmission strain gauges were used to calculate the strain and stress induced in the muscle specimen.

\subsection{Experimental Protocol}

A total 40 tubular muscle specimens (20 along the fiber direction and 20 along the perpendicular fiber direction) prepared from lower extremity muscles of the caprine were testes under dynamic tensile loading at four strain rates of $500 \mathrm{~s}^{-1}, 1000 \mathrm{~s}^{-1}, 1700 \mathrm{~s}^{-1}, 2700 \mathrm{~s}^{-1}$ and $3500 \mathrm{~s}^{-1}$ in each direction along and perpendicular to the muscle fiber orientation. At each strain rate, the tests were repeated at least 4 times under identical loading conditions to study the repeatability of experimental results. The only one test was conducted on each sample. For every test, the tubular muscle specimen was clamped between the incident bar and transmitter bar using plastic snap clamps. All the tests were performed till failure of specimen in unconfined condition. The unconfined condition means the specimens were allowed to enlarge freely in the longitudinal direction till failure and contract in the lateral direction. All the experimental tests were conducted at room temperature $\left(20-25^{\circ} \mathrm{C}\right)$.

\subsection{Data Analysis}

LabVIEW assisted data acquisition system composed of
NI 9237 bridge module and NIcDAQ 9174 with LabVIEW 2014 software has been established to acquire and records the stress wave history using strain gauges mounted on the pressure bars. The incident and transmission bar signals were captured using incident and transmission strain gauges at $50 \mathrm{kHz}$ for 4 milliseconds resulting in 200 data points per test. During processing, the signal conditioning and amplification was done using continuous sampling acquisition mode to make signal suitable for processing. The waveform data was filtered at $10 \mathrm{kHz}$ during data acquisition using low pass filter for the reduction of noise. The signal to noise ratio (SN ratio) was evaluated for the acquired waveforms and tests with SN ratio lower than 10 were excluded for further analysis [22]. The MATLAB program based on viscoelastic analysis was used to post process the recorded waveform. This enables the calculation of the propagation coefficient, reconstruction of the waveform at specimen-bar interface and determination of the stresses, strains and strain rates in the muscle specimen to plot the stress-strain response at each strain rate.

\section{Results and Discussion}

An experimental approach as suggested by Bacon [32] has been implemented for the viscoelastic analysis of polymeric SHPB and it was found that phase velocity increases with increase in frequency. The forces at the free end form the measured strains has been calculated using elastic and viscoelastic analysis of SHPB as suggested by to validate the test methodology [32]. By using elastic analysis, forces were calculated at the free end $\left(\mathrm{F}_{\text {elastic }}\right)$ from the strains measured at strain gauge mounted location. In this method, the phase velocity $c(\omega)$ was considered to be independent of the frequency and the attenuation coefficient $\alpha(\omega)$ was assumed to be zero at all frequencies implying that there was no attenuation and dispersion in the waves across its travel, in other words the bar material was considered to be pure elastic. To implement this condition, phase velocity c $(\omega)$ was kept constant at all frequencies while calculating the wave number $\mathrm{k}(\omega)$. In the second method, viscoelastic analysis was performed to calculate the forces $\left(\mathrm{F}_{\text {visco }}\right)$ at the free end by considering the attenuation and dispersion in waves from the measured strain gauge location. Therefore, the forces at the free end were calculated from the strains measured at gauge location by subjecting the propagation distance as $d=610 \mathrm{~mm}$. Hence, this analysis considers the frequency dependency of the phase velocity and attenuation coefficient. The forces obtained are then compared in the figure 5 and it was found that the forces at the free end determined using viscoelastic analysis is nearly zero. This validates the polymeric SHPB and can be implemented for soft tissue characterization. It has also been observed that the maximum force of $40 \mathrm{~N}$ developed at free end due to elastic analysis and zero force is 
developed at free end due to viscoelastic analysis. An elastic analysis will give erroneous results if elastic analysis is used for the determination of stress-strain response for the SHPB with viscoelastic pressure bars. Hence, elastic cannot be directly used for viscoelastic SHPB with polymeric bars.

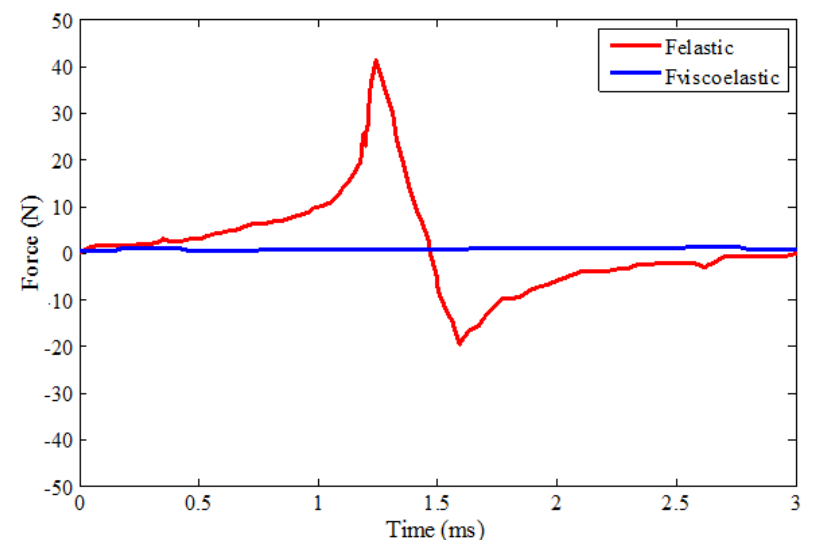

Figure 5. Forces determined at free end of bar using elastic and viscoelastic analysis of SHPB

The typical time histories of strain and strain rate of muscle tissue at strain rate of $2700 \mathrm{~s}^{-1}$ is shown in figure 6 . The nearly flat top portion indicates that the muscle specimen deformed at a nearly constant strain rate

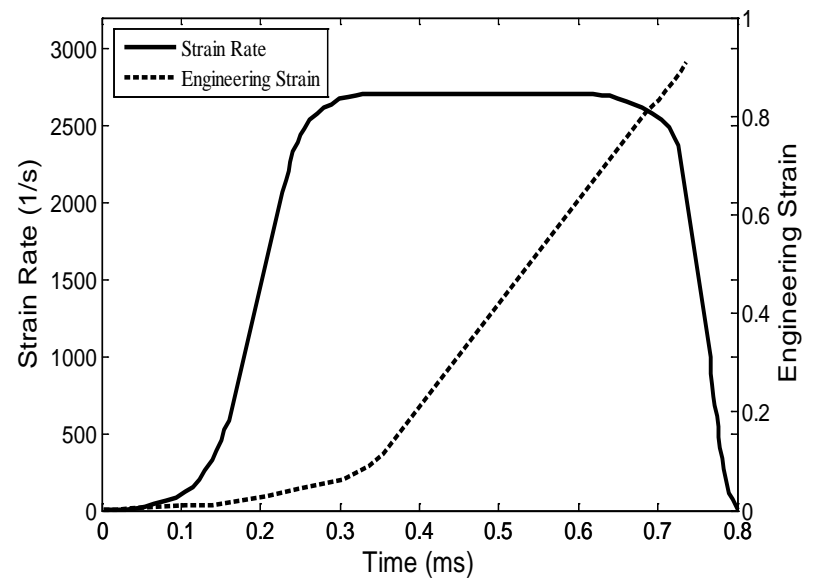

Figure 6. Strain and strain histories in the caprine muscle specimen

The resultant average tensile stress-strain curves are depicted in figures 7 and figure 8 for the loading directions along and perpendicular to the muscle fiber direction respectively. Each muscle specimen was tested at five impact velocities corresponding to five strain rates form $500-3500 \mathrm{~s}^{-1}$ up to failure. Each test was repeated at least four times to obtain stress-strain curves at similar loading conditions and each muscle specimen was tested once to the point of failure to investigate the effects of rate dependence. It is noted that each of the curve in the two figures represent the average of at least four repeated experimental tests performed under same loading conditions. The response of caprine muscle tissue was non-linear in tensile loading for all loading rates.

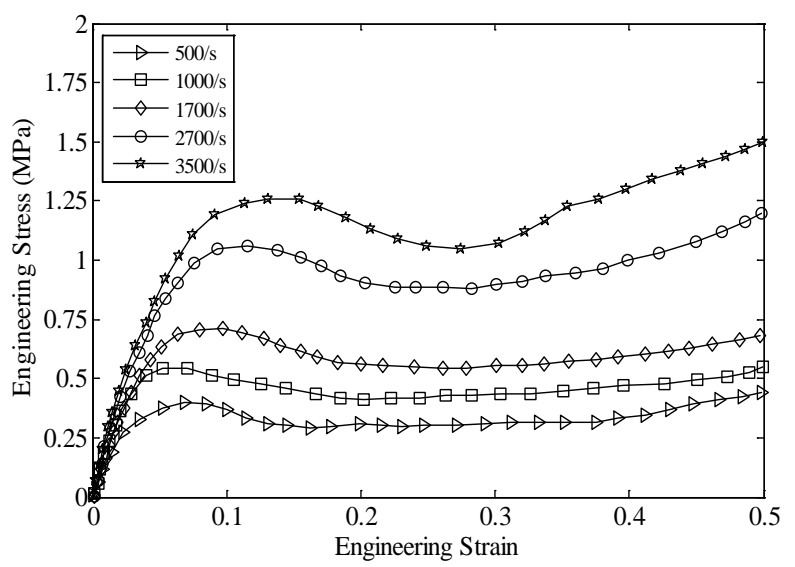

Figure 7. Average stress-strain curves of the caprine muscle along the fiber direction at various strain rates

The results of the current study also showed that the response of muscle tissue varied with respect to higher dynamic loading rates, with higher rate tests giving higher failure stresses and higher failure strains. The increase in applied strain rate leads to the increase in the work hardening effect of flow stress. There is absence of toe region at the beginning which indicates that the muscle fibers are straight and smooth at the start of the loading. The increase in applied strain rate leads to the increase in the work hardening effect of flow stresses. The stress-strain curves shows that the muscle tissue response is sensitive to both strain and strain rate. For both fiber direction cases, the steep initial rise is considered to be first phase in the stress-strain response. In the second phase, strain starts to accumulate rapidly without profound increase in stress up to failure. The similar trends of tensile stress-strain curves were reported in previous studies on porcine muscle at quasi-static and high strain rates [30] and human muscles at intermediate strain rates [10].

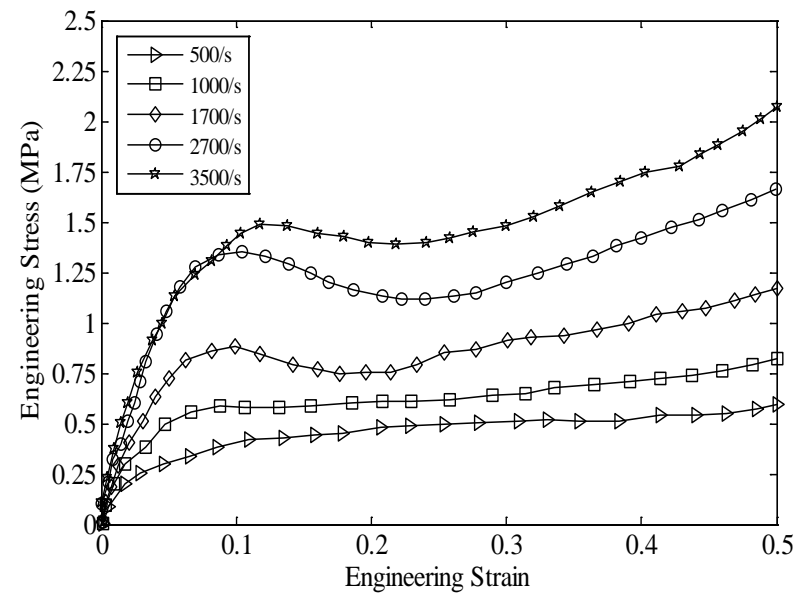

Figure 8. Average stress-strain curves of the caprine muscle perpendicular to the fiber direction at various strain rates 
Authors were not studied the quasi-static response of the caprine muscle. The specimens used in current study were approximately similar to those as used in Nie et al. [24] study. It was found that the stress-strain response obtained in previous studies [24, 34] at quasi-static loading showed a similar nature when compared with current SHPB response at dynamic loading. Most of the previous studies show that the muscle behavior is viscoelastic [1, 35-36] and viscoelasticity displayed during tensile loading is likely due to the passive properties of protein titin [37-38]. But, very few studies observed the muscle behavior at varying strain rates. The results of the present study show that the stress-strain response is explicitly dependent on strain rates applied. It is also observed that at the same strain rate, the specimen stress perpendicular to fiber direction (TR) was higher than that along the fiber direction (AL). This may be due to different force production mechanisms for the muscle along and perpendicular to the fiber direction. Muscle fibers are made from myofibrils. Myofibrils are composed of sarcomeres arranged in series and each sarcomere has overlapping myosin and actin filaments. The fiber-shaped fascicles and perimysium becomes the load bearing element if the muscle is loaded along the fiber direction and perpendicular to the fiber direction respectively. The stretch-resisting characteristic of perimysium may result in the higher deformation in the perpendicular muscle fiber direction [32].

Table 1. Stress values at various strain rates observed in caprine muscle along and perpendicular to muscle fiber direction at $40 \%$ strain

\begin{tabular}{|c|c|c|}
\hline \multirow{2}{*}{ Strain Rate $\left(\mathrm{s}^{-1}\right)$} & \multicolumn{2}{|c|}{ Stress (MPa) at 40\% Strain } \\
\cline { 2 - 3 } & $\mathrm{AL}$ & $\mathrm{TR}$ \\
\hline 500 & 0.3 & 0.5 \\
\hline 1000 & 0.45 & 0.7 \\
\hline 1700 & 0.6 & 1 \\
\hline 2700 & 1 & 1.4 \\
\hline 3500 & 1.3 & 1.75 \\
\hline
\end{tabular}

At $40 \%$ strain, the values of stress at various strain rates are shown in table 1 . At $40 \%$ strain, a maximum stress of 1.3 MPa and 1.75 MPa have been observed in caprine muscle loaded along and perpendicular to the fiber direction respectively at a strain rate of $3500 \mathrm{~s}^{-1}$. Nie et al. [21] have reported a maximum stress of $0.3 \mathrm{MPa}$ and 0.35 MPa at 30\% strain for the porcine muscle loaded along and perpendicular to fiber direction at $2100 \mathrm{~s}^{-1}$. The similar trend of stress-strain curve in regard to the loading rate is observed in the literature on tensile testing of porcine muscle [24] and pig skin [28] at higher strain rates using polymeric SHPB. The caprine muscle response in the present study has been found to be stiffer than porcine muscle response reported by Nie et al. [30]. The tensile stress-strain response of caprine muscle tissue was found to be higher than that of porcine muscle tissue.

\section{Conclusions}

The uniaxial tensile stress-strain response of caprine (goat) muscle has been determined experimentally at varying strain rates ranging from 500 to $3500 \mathrm{~s}^{-1}$ using custom-made polymeric SHPB. The muscle tissues were harvested from lower extremity of caprine and thin annular specimens were prepared along and perpendicular to the fiber direction. The experimental stress-strain responses are found to be non-linear and highly dependent on strain rate. The absence of toe regions on tensile stress-strain curves indicates that muscle fibers are straight and smooth at the start of loading. The maximum tensile stresses and strains increase significantly with increase in strain rates. It is also observed that at the same strain rate, the specimen stress of caprine muscle along the perpendicular fiber (TR) direction is higher than that along the fiber (AL) direction. The use of polymeric bars in SHPB is suitable for characterization of muscle tissue at high strain rates. Experimental stress-strain data under tensile loading can be used in computational modeling of human body for impact based simulations of high rate scenarios.

\section{Acknowledgements}

The authors would like to acknowledge DBATU, Lonere for providing the research facility in the department of mechanical engineering. The authors are also thankful to M/S Shree Engineering, Baramati for providing technical support to develop customized SHPB setup and ARK Info-solutions Pvt. Ltd., Pune for their help during experiments.

\section{Abbreviations / Nomenclature}
NI
SHPB
PMHS
$\varepsilon_{i}$
$\varepsilon_{r}$
$\varepsilon_{t}$
$\varepsilon_{S}(t)$
$\dot{\varepsilon}_{S}(t)$
National Instruments
$\sigma_{S}(t)$
$V_{s t}$
$A_{S}$
$L_{s}$
Split Hopkinson Pressure Bar
Post Mortem Human Subject
Incident pulse
Reflected pulse
Transmitted pulse
Strain induced in specimen
Strain rate induced in specimen
Stress induced in specimen
Impact velocity of striker bar
Initial cross-sectional area of specimen
Initial length of specimen
Length of pressure bar 


$\begin{array}{ll}\gamma(\omega) & \begin{array}{l}\text { Propagation coefficient at frequency } \omega \\ E^{*}(\omega)\end{array} \\ \tilde{\varepsilon}_{R_{-} \text {new }}(\omega) & \begin{array}{l}\text { Remplex Young's modulus of the bar } \\ \text { frequency }\end{array} \\ \tilde{\varepsilon}_{T} \_ \text {new }(\omega) & \begin{array}{l}\text { Reconstructed transmitted strain at } \\ \text { frequency } \omega\end{array} \\ \varepsilon_{S}(t) & \text { Strain in the specimen at frequency } \omega \\ \dot{\varepsilon}_{S}(t) & \text { Strain rate in specimen at frequency } \omega \\ \sigma_{S}(\omega) & \begin{array}{l}\text { Average stress in the specimen at } \\ \text { frequency } \omega\end{array} \\ \mathrm{c}(\omega) & \begin{array}{l}\text { Phase velocity } \\ \alpha(\omega)\end{array} \\ \mathrm{k}(\omega) & \begin{array}{l}\text { Attenuation coefficient } \\ \text { Wave number }\end{array} \\ \mathrm{F}_{\text {elastic }} & \begin{array}{l}\text { Force due to elastic analysis by Bacon } \\ \text { approach }\end{array} \\ \mathrm{F}_{\text {visco }} & \begin{array}{l}\text { Force due to viscoelastic analysis by } \\ \text { Bacon approach }\end{array}\end{array}$

\section{REFERENCES}

[1] L. Van, C. Lyons, C. Simms. Viscoelastic properties of passive skeletal muscle in compression: stress-relaxtaion behaviour and constitutive modeling, Journal of Biomechanics, Vol. 41, 1555-1566, 2008.

[2] H. Warhatkar, A. Chawla, S. Mukherjee, R. Malhotra. Experimental Study of Variation between Quasi-Static and Dynamic Load Deformation Properties of Medial Collateral Knee Ligaments, SAE World Congress, Detriot, 2009.

[3] X. Zhai, W. Chen. Compressive Mechanical Response of Porcine Muscle at Intermediate $\left(10^{0} / \mathrm{s}-10^{2} / \mathrm{s}\right)$ Strain Rates, Experimental Mechanics, 2018.

[4] X. Zhai, E. Nauman, Y. Nie, H. Liao et al. Mechanical response of human muscle at intermediate strain rates, Journal of Biomechanical Engineering, 2019.

[5] A. R. Kemper, A. C. Santago, J. D. Stitzel, J. L. Sparks and S. M. Duma. Biomechanical response of human spleen in tensile loading. Journal of Biomechanics, Vol. 45, No. 2, 348-355, 2012.

[6] P. Gaur, A. Chawla, K. Verma, S. Mukherjee, S. Lalvani, R. Malhotra, and C. Mayer. Characterization of human diaphragm at high strain rate loading, Journal of the Mechanical Behavior of Biomedical Materials, Vol. 60, 603-616, 2016.

[7] D. Mohan, J. W. Melvin. Failure properties of passive human aortic tissue. I-Uniaxial tension tests, Journal of Biomechanics, Vol. 15, No. 11, 887-902, 1982.

[8] J. Hopkinson. Further experiments on the rupture of iron wire, Article 39, B. Hopkinson (ed.) (1872), Original Papers-by the late John Hopkinson, Vol. II, Scientific Papers, Cambridge, 1901.
[9] B. Hopkinson. A Method of Measuring the Pressure Produced in the Detonation of High Explosives or by the Impact of Bullets, Philosophical Transactions of the Royal Society of London: Series A, Vol. 213, 437-456, 1914.

[10] R. Davis. A critical study of the Hopkinson pressure bar, Philosophical Transactions of the Royal Society of London: Series A, Vol. 240, 375-457, 1948.

[11] H. Kolsky. An investigation of the mechanical properties of materials at very high rates of loading, Proceedings of the Physical Society-Section B 62, 676-700, 1949.

[12] E. Davies, S. Hunter. The Dynamic Compression Testing of Solids by the Method of the Split Hopkinson Pressure Bar, Journal of the Mechanics and Physics of Solids, Vol. 11, No. 3, 155-179, 1963.

[13] F. Pervin, W. Chen, T. Weerasooriya. Dynamic compressive response of renal cortex, International Journal of Structural Changes in Solids, Vol. 2, No. 1), 1-7, 2010.

[14] F. Pervin, W. Chen, T. Weerasooriya. Dynamic compressive response of bovine liver tissue, Journal of Mechanical Behavour Biomedical Materials, Vol. 4, 76-84, 2011.

[15] F. Pervin, W. Chen. Dynamic mechanical response of bovine gray and white matter brain tissues under compression, Journal of Biomechanics, Vol. 42, 731-735, 2009.

[16] J. Chen, S. Patnaik, R. Prabh, L. Proddy et al. Mechanical response of porcine liver tissue under high strain rate compression, Bioengineering, Vol. 6, No. 49, 1-16, 2019.

[17] K. Comley, N. Fleck. The compressive response of porcine adipose tissue from low to high strain rate, International Journal of Impact Engineering, Vol. 46, 1-10, 2012.

[18] B. Butler, C. Bo, A. Tucker, A. Jardine et al., Mechanical and histological characterization of trachea tissue subjected to blast-type pressures, IOP Journal of Physics: Conference Series, 18 ${ }^{\text {th }}$ APS-SCCM and 24th AIRAPT, 500, 2014.

[19] B. Sanborn, X. Nie, W. Chen, T. Weerasooriya. High strain-rate pure shear and uniaxial compressive response of porcine lung tissue, Journal of Applied Mechanics, Vol. 80, 11-29, 2013.

[20] B. Song, W. Chen, Y. Ge, T. Weerasooriya. Dynamic and quasi-static compressive response of porcine muscle, Journal of Biomechanics, Vol. 40, 2999-3005, 2007.

[21] S. C. Van, D. S. Cronin, G. Brodland. High strain rate compressive properties of bovine muscle tissue determined using split Hopkinson bar apparatus, Journal of Biomechanics, Vol. 39, 1852-1858, 2006.

[22] B. Karthikeyan, S. Mukherjee, A. Chawala, R. Malhotra, Dynamic compressive response of passive human muscles using Split Hopkinson Pressure Bar, Indian Journal of Biomechanics, Vol. 1, No. 3, 20-28, 2012.

[23] S. H. Kadhane, H. N. Warhatkar. Dynamic Stress-Strain Compressive Response of Soft Tissue using Polymeric Split Hopkinson Pressure Bar, International Journal of Innovative Technology and Exploring Engineering, Vol. 8, No. 9, 341-347, 2019.

[24] X. Nie, J. Cheng, W. Chen, T. Weerasooriya. Dynamic tensile response of porcine muscle, Journal of Applied Mechanics, Vol. 78, No. 2, 021009-1-021009-5, 2011. 
[25] B. Z. Wang, Y. X. Zhen, S. S. Hu. Dynamic tensile properties of porcine ham muscle, Explosion and Shock Waves, Vol. 30, 33-38, 2010.

[26] M. Cheng, W. Chen, T. Weerasooriya. Mechanical behavior of bovine tendon with stress and loading rate effects, Advanced Theoretical Applied Biomechanics, Vol. 2, No.2, 59-74, 2009.

[27] O. Shergold, N. Fleck, D. Radford. The uniaxial stress versus strain response of pig skin and silicone rubber at low and high strain rates, International Journal of Impact Engineering, Vol. 32, 1384-1402, 2006.

[28] J. Lim, J. Hong, W. Chen, T. Weerasooriya. Mechanical response of pig skin under dynamic tensile testing, International Journal of Impact Engineering, Vol. 38, 130-135, 2011.

[29] V. P. W. Shim, J. F. Liu, V. S. Lee. A technique for dynamic tensile testing of ligaments from the human cervical spine ligaments, Experimental Mechanics, Vol. 46, 77-89, 2006.

[30] M. Trexler, A. Lennon, A. Wickwire, T. Harrigan. et al. Verification and implementation of a modified split Hopkinson pressure bar technique for characterizing biological tissue and soft biosimulant materials under dynamic shear loading, Journal of Mechanical Behavior of Biomedical Materials, Vol. 4, 1920-928, 2011.

[31] W. Chen. Experimental methods for characterizing dynamic response of soft materials, Journal of Dynamic Behavior of Materials, Vol. 2, 2-14, 2016.
[32] C. Bacon. An experimental method for considering dispersion and attenuation in a viscoelastic Hopkinson bar, Experimental Mechanics, Vol. 38, 242-249, 1998.

[33] S. H. Kadhane, H. N. Warhatkar. Mechanical Response of Caprine Muscle under Compression at Varying Strain Rates. Test Engineering and Management, Vol. 82, 1245-1252, 2020.

[34] G. Mutungi, P. Purslow, C. Warkup. Structural and Mechanical Changes in Raw and Cooked Single Porcine Muscle Fibers Extended to Fracture, Meat Science, Vol. 40, 217-234, 1995.

[35] T. M. Best, J. MacElhaney, E. G. William, B. S. Mayers. Characterization of passive response of live skeletal muscle using quasi-linear theory of viscoelasticity. Journal of Biomechanics. Vol. 27, 413-419, 1994.

[36] G. A. Meyer, A. D. McCulloch, R. L. Lieber. A nonlinear model of passive muscle viscosity. Journal of Biomechanical Engineering, Vol. 133, 091007, 2011.

[37] K. Wang, R. McCarter, J. Wright, J. Beverly, R. Ramirez-Mitchell. Viscoelasticity of the sarcomere matrix of skeletal muscles: The titin-myosin composite filament is a dual-stage molecular spring. Biophys Journal, Vol. 64, 1161-1177, 1993.

[38] M. L. Bartoo, W. A. Linke, G. H. Pollack. Basis of passive tension and stiffness in isolated rabbit myofibrils. American Journal of Physiology, Vol. 273, C266-C276, 1997. 